\title{
Observation of Heteronuclear Feshbach Molecules from $\mathbf{a}^{85} \mathrm{Rb}-{ }^{87} \mathrm{Rb}$ gas
}

\author{
S. B. Papp* and C. E. Wieman \\ JILA, National Institute of Standards and Technology and University of Colorado, Boulder, Colorado 80309-0440, USA
}

(Dated: August 27, 2018)

\begin{abstract}
We report on the observation of ultracold heteronuclear Feshbach molecules. Starting with a ${ }^{87} \mathrm{Rb} \mathrm{BEC}$ and a cold atomic gas of ${ }^{85} \mathrm{Rb}$, we utilize previously unobserved interspecies Feshbach resonances to create up to 25,000 molecules. Even though the ${ }^{85} \mathrm{Rb}$ gas is non degenerate, we observe a large molecular conversion efficiency due to the presence of a quantum degenerate ${ }^{87} \mathrm{Rb}$ gas; this represents a key feature of our system. We compare the molecule creation at two different Feshbach resonances with different magnetic-field widths. The two Feshbach resonances are located at $265.44 \pm 0.15 \mathrm{G}$ and $372.4 \pm 1.3 \mathrm{G}$. We also directly measure the small binding energy of the molecules through resonant magnetic-field association.
\end{abstract}

The creation of ultracold molecules from ultracold atoms is currently a topic of great experimental and theoretical interest [1, 2, 3, 4]. Ultracold heteronuclear molecules in low-lying vibrational states are particularly interesting since they are predicted to exhibit a permanent dipole moment due to the unequal distribution of electrons. Numerous proposals for utilizing polar molecules exist, including quantum computation [5] and the search for the electron electric dipole moment [6]. Although no significant permanent dipole moment is expected to exist in a ${ }^{85} \mathrm{Rb}-{ }^{87} \mathrm{Rb}$ molecule, our work demonstrates a first step toward the efficient production of ground-state ultracold heteronuclear molecules.

To date cold heteronuclear molecules in high-lying vibrational levels have been created using photoassociation 7, 8, 9]. These molecules can then be pumped toward low-lying vibrational levels by exciting boundbound molecular transitions [3] via, for example, stimulated Raman-type transitions [5] that enhance the probability of populating the lowest vibrational level. The initial photoassociation step used in this process is inefficient, and many final vibrational levels of the molecule are occupied. An alternative to this initial photoassociation step is the direct conversion of two free atoms into a molecule in the highest vibrational levels using a Feshbach resonance [1, 2, 10, 11, 12, 13, 14, 15]. High conversion efficiency using a Feshbach resonance has been demonstrated in single-species gases via adiabatic magnetic-field sweeps across the resonance [4, 16], threebody recombination [13], resonant magnetic-field association [15], and non adiabatic magnetic-field sweeps [1, 17]. Feshbach resonances between two different atomic species [18, 19] have previously been reported. Our work builds upon these observations by demonstrating stable heteronuclear Feshbach molecules.

In this Letter we present a systematic study of the creation of heteronuclear molecules from an atomic gas of ${ }^{85} \mathrm{Rb}$ and ${ }^{87} \mathrm{Rb}$. We find that these molecules can be created using the standard techniques already proven with single-species gases. The presence of two species with different quantum degeneracy provides a rich system for testing our understanding of the conversion efficiency from atoms to molecules. Furthermore, the molecule creation process allows us to determine the location and width of Feshbach resonances in the two-species system; this information will be required for future studies of the ${ }^{85} \mathrm{Rb}-{ }^{87} \mathrm{Rb}$ system with a tunable interspecies interaction.

Our apparatus is designed to create a two-species Bose gas through sympathetic cooling of ${ }^{85} \mathrm{Rb}$ with ${ }^{87} \mathrm{Rb}$. The details of our system are similar to those described in Refs. 20, 21] and are only briefly described here. We initially collect approximately $3 \times 10^{9}{ }^{87} \mathrm{Rb}$ atoms and $10^{7}$ ${ }^{85} \mathrm{Rb}$ atoms in a two-species vapor cell magneto-optical trap (MOT). The atoms are loaded into a quadrupole magnetic trap with a $100 \mathrm{G} / \mathrm{cm}$ magnetic-field gradient in the axial direction of the coils and physically transported to another region of the apparatus with lower vacuum pressure 20, 22]. Here the atoms are prepared in the ${ }^{85} \mathrm{Rb}\left|f=2, m_{f}=-2\right\rangle$ state and ${ }^{87} \mathrm{Rb}\left|f=1, m_{f}=-1\right\rangle$ state and loaded into a Ioffe-Pritchard-type magnetic trap. Selective rf evaporation is used to lower the temperature of the ${ }^{87} \mathrm{Rb}$ gas, while the ${ }^{85} \mathrm{Rb}$ gas is sympathetically cooled through thermal contact with the ${ }^{87} \mathrm{Rb}$ [23].

The mixed-species gas is evaporatively cooled in the magnetic trap to approximately $10 \mu \mathrm{K}$ and then further evaporatively cooled in an optical dipole trap. The optical trap is formed at the focus of a Yb:YAG laser beam with a $1 / e^{2}$ radius of $23.5 \mu \mathrm{m}$. To evaporate in the optical trap the power is lowered from $1.2 \mathrm{~W}$ to $14 \mathrm{~mW}$ in 10 seconds. This trap has the distinct advantage of holding any spin state in the same spatial location while allowing a variable magnetic field to be applied. A Helmholtz pair of coils provides a magnetic field up to $700 \mathrm{G}$; the magnetic field is calibrated using rf-driven Zeeman transitions with a systematic uncertainty of $0.01 \%$. We perform the optical trap evaporation slightly above the ${ }^{85} \mathrm{Rb}$ Feshbach resonance at $155 \mathrm{G}$ where density-dependent ${ }^{85} \mathrm{Rb}-{ }^{85} \mathrm{Rb}$ loss is minimized [24]. We typically produce a ${ }^{87} \mathrm{Rb}$ BEC with 300,000 atoms and 50\% condensate fraction and a non degenerate $\left(\mathrm{T} / \mathrm{T}_{c}=2.4\right)$ gas of ${ }^{85} \mathrm{Rb}$ with 40,000 atoms. Measurements are performed in an optical trap with a radial trap frequency of $\omega_{r}=2 \pi \times 200$ 


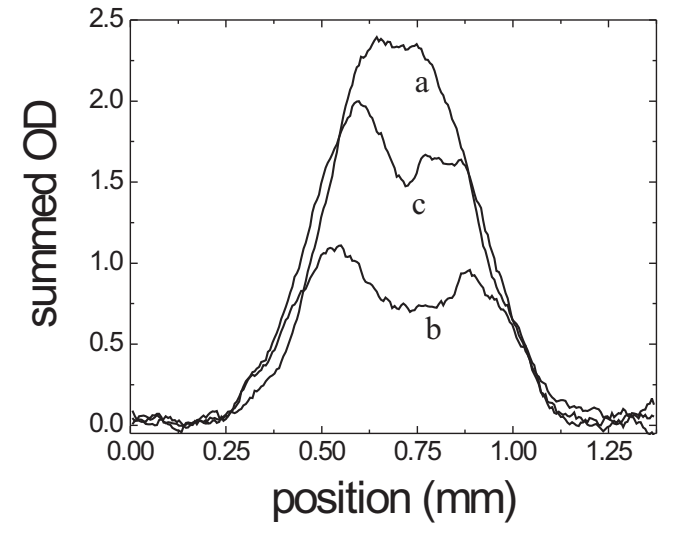

FIG. 1: Absorption-image axial cross sections of the ${ }^{85} \mathrm{Rb}$ gas demonstrating reversible molecule creation. The measured two-dimensional optical density (OD) was summed in the remaining radial direction of the absorption image. (a) Prior to sweeping the magnetic field the atom number is 26,500. (b) After sweeping through the resonance $53 \%$ of the gas is converted to molecules. (c) By reversing the molecule creation process approximately $85 \%$ of the initial atom number is observed to reappear. Note that most of the ${ }^{85} \mathrm{Rb}$ loss occurs in the center of the gas where the ${ }^{87} \mathrm{Rb} \mathrm{BEC}$ density is largest.

$\mathrm{Hz}$ and an aspect ratio of approximately 100 .

We have discovered two heteronuclear $s$-wave Feshbach resonances in the ${ }^{85} \mathrm{Rb}\left|f=2, m_{f}=-2\right\rangle$ state and ${ }^{87} \mathrm{Rb}\left|f=1, m_{f}=-1\right\rangle$ state, one near $265 \mathrm{G}$ and the other near $372 \mathrm{G}$. The binding energy of the bound molecular state increases with magnetic field above the position of each Feshbach resonance. To create heteronuclear molecules we adiabatically $(450 \mu \mathrm{s} / \mathrm{G})$ sweep [2] the magnetic field from low to high field through a Feshbach resonance. Figure 1b shows an absorption-image cross section of the ${ }^{85} \mathrm{Rb}$ gas following a sweep through the resonance. Prior to imaging the gas is released from the optical trap and expands for $3 \mathrm{~ms}$; at the same time as the optical trap release is initiated, the magnetic field is switched off within $50 \mu \mathrm{s}$. A significant fraction $(\sim 60 \%)$ of the atoms disappear as compared to an absorptionimage cross section without sweeping the magnetic field (Fig. 19). When we reverse the molecule creation process by immediately applying a second field sweep in the opposite direction less than $100 \mu$ s after the first sweep ends, (Fig. 1:), a large fraction of the atoms reappear [2]. The atoms that reappear represent reversible heteronuclear molecule formation. The small fraction of atoms that do not return are lost to ${ }^{85} \mathrm{Rb}-{ }^{87} \mathrm{Rb}$ inelastic collisions that remove atoms from the trap. We verified this by sweeping across the resonance at the same rate in the opposite direction. At a typical ${ }^{87} \mathrm{Rb}$ peak density of $1 \times 10^{14} \mathrm{~cm}^{-3}$ we observe approximately $15 \%{ }^{85} \mathrm{Rb}$ num-
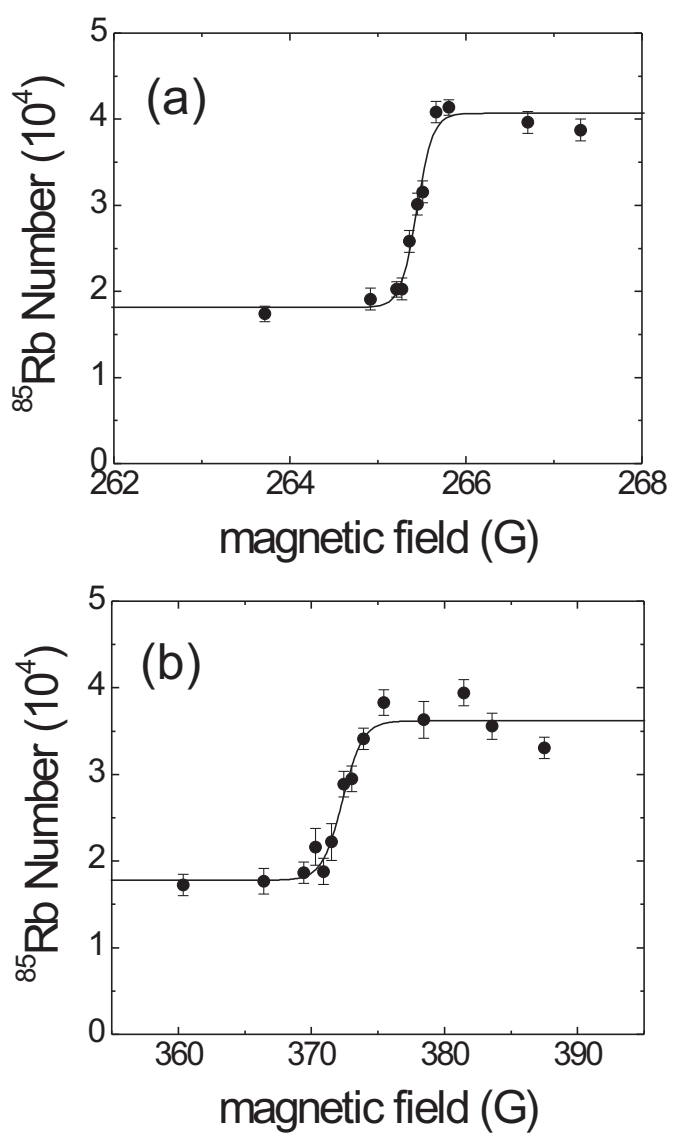

FIG. 2: Atom loss after magnetic-field sweeps through a Feshbach resonance. The number of ${ }^{85} \mathrm{Rb}$ atoms is a function of the final magnetic field during the sweeps near the (a) 265 $\mathrm{G}$ and (b) $372 \mathrm{G}$ Feshbach resonances. Initially there are $2.1-2.3 \times 10^{5}{ }^{87} \mathrm{Rb}$ atoms at $\mathrm{T} / \mathrm{T}_{c}$ between 0.82 and 0.84 . The data are fitted to an error function to extract the center position and width. The resulting positions of the two transitions are $265.44 \pm 0.15 \mathrm{G}$ and $372.4 \pm 1.3 \mathrm{G}$ with the uncertainty given by the fitted RMS width.

ber loss during a sweep. By monitoring the reappearance of atoms as a function of time, we find that the molecules decay with a lifetime of approximately $1 \mathrm{~ms}$. This decay is due to a combination of inelastic collisions with the ${ }^{87} \mathrm{Rb}$ gas and a one-body spontaneous process [25, 26].

We precisely located the Feshbach resonances with an experimental technique that avoids the need for rapid magnetic-field sweeps. At the lower (higher) field resonance we sweep the magnetic field from 269 G (397 G) downward toward the resonance stopping at various final values. The rate of this magnetic field sweep is fast compared to both the timescale for molecule creation and atom loss due to inelastic collisions. The field is held at 
the final value for $0.3 \mathrm{~ms}$ and then returns to $269 \mathrm{G}$ (397 $\mathrm{G})$ at a rate of $450 \mu \mathrm{s} / \mathrm{G}(70 \mu \mathrm{s} / \mathrm{G})$ which is slow compared to the molecule creation rate. The magnetic field remains here for $5 \mathrm{~ms}$ to ensure that any molecules made during the second sweep decay and are lost from the optical trap [25, 26]. We then simultaneously turn off the magnetic field and the optical trap to let the gas expand for $6 \mathrm{~ms}$ and measure the number of ${ }^{85} \mathrm{Rb}$ atoms remaining. In Fig. 2 we show the atom number remaining as a function of the final magnetic field for the two Feshbach resonances. The key to this method is that if the field passes through the Feshbach resonance on the first sweep, then a fraction of the atoms will be converted into molecules by the second sweep. The rapid onset of atom loss due to molecule creation when the magnetic field is swept below $265.44 \mathrm{G}(372.4 \mathrm{G})$ represents crossing the peak of the Feshbach resonance. A significant fraction ( 50\%) of the ${ }^{85} \mathrm{Rb}$ gas is converted to molecules.

The dependence of molecule conversion efficiency on sweep rate was measured in Ref. [16] and is well characterized by a Landau-Zener model. For our two-species gas with similar number and temperature we observe a factor of $5.3 \pm 1.9$ decrease in the ramp rate required to create molecules at the $372 \mathrm{G}$ Feshbach resonance as compared to the $265 \mathrm{G}$ resonance. This factor is consistent with the ratio of the predicted widths of the two resonances, which is in the range of $6-8$ [27, 28, 29]. This directly verifies the predicted inverse relationship between the Feshbach resonance width and the sweep rate required to create molecules.

We have investigated the adiabatic conversion efficiency of atoms to molecules. We begin by sweeping the magnetic field upward through the $265 \mathrm{G}$ Feshbach resonance at a rate that is slow with respect to the molecule conversion rate. It is then held at $269 \mathrm{G}$ for $5 \mathrm{~ms}$ to allow the molecules to decay and be lost from the trap. The number of molecules formed is simply the difference in ${ }^{85} \mathrm{Rb}$ number before and after the sweep with a small correction applied to account for measured inelastic atom loss during the field sweeps. The conversion efficiency to heteronuclear molecules is shown in Fig. 3 as a function of ${ }^{87} \mathrm{Rb} \mathrm{T} / \mathrm{T}_{c}$. We varied $\mathrm{T} / \mathrm{T}_{c}$ by either changing the number of ${ }^{87} \mathrm{Rb}$ atoms or the temperature. The ${ }^{85} \mathrm{Rb}$ gas had a $\mathrm{T} / \mathrm{T}_{c}$ in the range of 2.2 to 4 . We observe up to $60 \%$ conversion of the ${ }^{85} \mathrm{Rb}$ gas into molecules even when the ${ }^{85} \mathrm{Rb}$ gas is far from quantum degeneracy. The largest conversion efficiency is observed when ${ }^{85} \mathrm{Rb}$ is least quantum degenerate, indicating that the conversion efficiency primarily depends on ${ }^{87} \mathrm{Rb} \mathrm{T} / \mathrm{T}_{c}$.

In a single-species gas the molecule conversion efficiency of an adiabatic field sweep was shown to depend only on the degree of quantum degeneracy [16, 30]. A molecule is formed during an adiabatic sweep if two free atoms are sufficiently close in phase space so that their wavefunction can smoothly evolve to a bound molecule as the Feshbach resonance is crossed. This model also

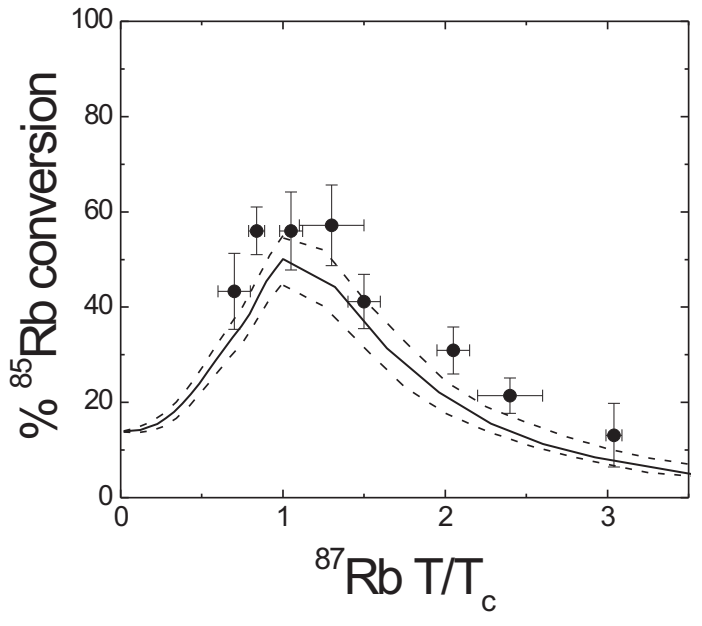

FIG. 3: Heteronuclear molecule conversion efficiency at the $265 \mathrm{G}$ Feshbach resonance as a function of ${ }^{87} \mathrm{Rb} \mathrm{T} / \mathrm{T}_{c}$. At our largest conversion efficiency the ${ }^{85} \mathrm{Rb}$ gas has $\mathrm{T} / \mathrm{T}_{c}=2.6$. The solid line shows a simulation based on our conversion model, and the dashed lines represent the uncertainty. The conversion drops below $\mathrm{T} / \mathrm{T}_{c}=1$ since the $\mathrm{BEC}$ is spatially smaller than the thermal ${ }^{85} \mathrm{Rb}$ gas and the conversion process depends on the proximity of two atoms in phase space.

explains the behavior we observe here. Each particle of the less degenerate species is surrounded in phase space by particles of the more degenerate ${ }^{87} \mathrm{Rb}$ gas with which molecules can be formed. We used a Monte-Carlo simulation to model the observed conversion efficiency. Phase space distributions of position and momenta of ${ }^{85} \mathrm{Rb}$ and ${ }^{87} \mathrm{Rb}$ are randomly generated based on the number and temperature of each gas and the trap frequencies. For the ${ }^{87} \mathrm{Rb}$ gas at temperatures above $\mathrm{T}_{c}$ a MaxwellBoltzmann (MB) distribution is used. Below $\mathrm{T}_{c}$ we use a combination of a Thomas-Fermi distribution for the BEC and a Bose-Einstein distribution for the thermal component. A MB distribution is always used for the ${ }^{85} \mathrm{Rb}$ gas since the temperature of the gas does not fall below $\mathrm{T}$ $=2.2 \mathrm{~T}_{c}$. For each ${ }^{85} \mathrm{Rb}$ atom the simulation searches the ${ }^{87} \mathrm{Rb}$ gas to find a pair that is sufficiently close in phase space. After a pairing occurs, the two atoms are removed from the simulation. Two atoms are considered sufficiently close in phase space if the following conditions are met: (1) If a BEC is present, any ${ }^{85} \mathrm{Rb}$ atoms inside the Thomas Fermi radius of the condensate form a molecule, and (2) outside the BEC, an ${ }^{85} \mathrm{Rb}$ atom and a partner ${ }^{87} \mathrm{Rb}$ atom must satisfy the relation found in Ref. [16]: $\left|\delta r_{r e l} m \delta v_{r e l}\right|<\gamma h$, where $\delta r_{r e l}$ is the separation of the pair, $m$ is the atomic mass, $\delta v_{r e l}$ is the relative velocity, and $\gamma=0.44 \pm 0.03$.

The results of our simulation are shown by the solid 
line in Fig. 3. For $\mathrm{T} / \mathrm{T}_{c}>1$ each gas has roughly the same spatial size in the optical trap, and therefore molecule conversion can occur anywhere in the gas as long as the local phase space criterion is met. At $\mathrm{T} / \mathrm{T}_{c}=1$ and below, a significant fraction of the ${ }^{87} \mathrm{Rb}$ atoms are part of the condensate, which is spatially small compared to the extent of the ${ }^{85} \mathrm{Rb}$ gas. If the ${ }^{85} \mathrm{Rb}$ gas were a BEC, our simulation would predict that the molecule conversion efficiency quickly approaches $100 \%$ as the $\mathrm{T} / \mathrm{T}_{c}$ of each gas drops below one. There is good agreement between the experiment and simulation in both the non degenerate and quantum degenerate regimes, suggesting that the pairing model in Ref. [16] is also applicable to heteronuclear molecule creation.

We created heteronuclear molecules using a small oscillating magnetic field to do spectroscopy on the Feshbach bound state. The oscillating field causes two atoms to bind together and form a molecule [15, 31]. We first ramp the magnetic field from $269 \mathrm{G}$ to a selected value between 266.2 and $267 \mathrm{G}$ in $0.3 \mathrm{~ms}$. The field modulation is then applied using the Helmholtz coil pair for $20 \mathrm{~ms}$ with a peak-to-peak amplitude between 0.6 and $1.0 \mathrm{G}$ at a frequency up to $40 \mathrm{kHz}$. Next the field is returned to $269 \mathrm{G}$ and held there for $5 \mathrm{~ms}$, allowing any molecules made during the modulation to decay. Finally we determine the number of atoms remaining as before. As in Ref. [15], we observe strongly enhanced atom loss at certain frequencies, and the loss depends on the duration and amplitude of the modulation. A typical loss spectrum is shown in the inset of Fig. 4 the frequency at which we observe maximum atom loss gives a measure of the binding energy of the molecules 32 .

The resonant frequency of maximum atom loss is shown in Fig. 4 for various magnetic fields near the Feshbach resonance. For each loss curve the amplitude used converted roughly half the ${ }^{85} \mathrm{Rb}$ gas into molecules. The solid line is a fit to the data based on the universal form of the molecular binding energy near an $s$-wave Feshbach resonance [4]. In the fit the background scattering length is fixed to the value $213 \pm 7 a_{0}[33]$, and the Feshbachresonance peak position and width are varied; the best fit finds the peak position and width to be $265.42 \pm 0.08$ $\mathrm{G}$ and $5.8 \pm 0.4 \mathrm{G}$, respectively. These results are consistent with our previously discussed determination of the Feshbach-resonance peak position and with the predicted width of the Feshbach resonance in Refs. [27, 28, 29].

In summary, we have created heteronuclear Feshbach molecules from an ultracold gas of ${ }^{85} \mathrm{Rb}$ and ${ }^{87} \mathrm{Rb}$. We demonstrated that molecules can be produced with two methods, magnetic-field sweeps and resonant-field modulation. The conversion efficiency of ${ }^{85} \mathrm{Rb}$ into molecules can reach $60 \%$ even when that gas is not quantum degenerate. The heteronuclear molecules described here are ultracold and are stable for at least $1 \mathrm{~ms}$. These conditions may provide a first step toward the efficient production of ground-state heteronuclear molecules.

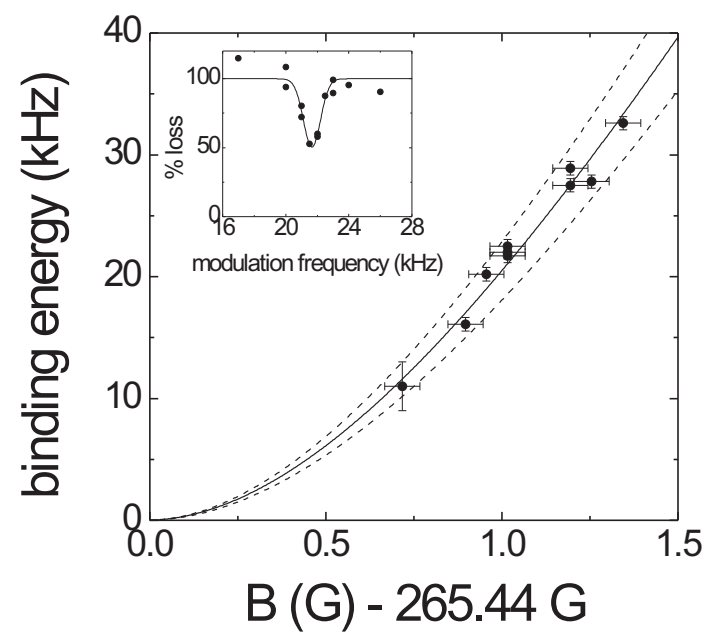

FIG. 4: Resonant frequency of atom loss as a function of magnetic field. The solid line is a fit to the data based on the universal binding energy of $s$-wave Feshbach molecules and the dashed lines represent the uncertainty in the Feshbach resonance width. (Inset) An atom-loss spectrum at $266.5 \mathrm{G}$ as a function of the modulation frequency. The modulation converts roughly $50 \%$ of the gas to molecules. The loss is centered at $21.7 \mathrm{kHz}$ with a width of $0.6 \pm 0.2 \mathrm{kHz}$. The solid line is a gaussian fit to the data. We report the uncertainty in the binding energy as the width of the loss spectrum because we lack a detailed understanding of the lineshape.

Recently evidence of heteronuclear molecule creation has been reported using ${ }^{40} \mathrm{~K}$ and ${ }^{87} \mathrm{Rb}$ in Refs. 34, 35].

We gratefully acknowledge useful discussions with Debbie Jin, John Bohn, Servaas Kokkelmans, and Cindy Regal. We thank Josh Zirbel and Juan Pino for experimental assistance. S. B. P acknowledges support from an NSF Graduate Fellowship. This work has been supported by NSF and ONR.

* Email: papp@jilau1.colorado.edu

[1] E. A. Donley, N. R. Claussen, S. T. Thompson, and C. E. Wieman, Nature 417, 529 (2002).

[2] C. A. Regal, C. Ticknor, J. L. Bohn, and D. S. Jin, Nature 424, 47 (2003).

[3] J. M. Sage, S. Sainis, T. Bergeman, and D. DeMille, Phys. Rev. Lett. 94, 203001 (2005).

[4] T. Köhler, K. Góral, and P. Julienne, cond-mat/0601420 (2006).

[5] D. DeMille, Phys. Rev. Lett. 88, 067901 (2002).

[6] J. J. Hudson, B. E. Sauer, M. R. Tarbutt, and E. A. Hinds, Phys. Rev. Lett. 89, 023003 (2002).

[7] A. J. Kerman et al., Phys. Rev. Lett. 92, 033004 (2004).

[8] D. Wang et al., Phys. Rev. Lett 93, 243005 (2004). 
[9] M. Mancini et al., Phys. Rev. Lett. 92, 133203 (2004).

[10] S. Dürr, T. Volz, A. Marte, and G. Rempe, Phys. Rev. Lett. 92, 020406 (2003).

[11] J. Herbig et al., Science 1088876 (2003).

[12] J. Cubizolles et al., Phys. Rev. Lett 91, 240401 (2003).

[13] S. Jochim et al., Phys. Rev. Lett. 91, 240402 (2003).

[14] K. E. Strecker, G. B. Partridge, and R. G. Hulet, Phys. Rev. Lett. 91, 080406 (2003).

[15] S. T. Thompson, E. Hodby, and C. E. Wieman, Phys. Rev. Lett. 95, 190404 (2005).

[16] E. Hodby et al., Phys. Rev. Lett. 94, 120402 (2005).

[17] N. R. Claussen, E. A. Donley, S. T. Thompson, and C. E. Wieman, Phys. Rev. Lett. 89, 010401 (2002).

[18] S. Inouye et al., Phys. Rev. Lett. 93, 183201 (2004).

[19] C. A. Stan et al., Phys. Rev. Lett. 93, 143001 (2004).

[20] H. Lewandowski, J. Low Temp. Phys. 132, 309 (2003).

[21] S. L. Cornish et al., Phys. Rev. Lett. 85, 1795 (2000).

[22] M. Greiner et al., Phys. Rev. Lett. 87, 160405 (2001).

[23] I. Bloch et al., Phys. Rev. A 64, 021402(R) (2001).

[24] J. L. Roberts, N. R. Claussen, S. L. Cornish, and C. E. Wieman, Phys. Rev. Lett. 85, 728 (2000).

[25] S. T. Thompson, E. Hodby, and C. E. Wieman, Phys. Rev. Lett. 94, 020401 (2005).

[26] T. Köhler, E. Tiesinga, and P. S. Julienne, Phys. Rev. Lett. 94, 020402 (2005).
[27] J. P. Burke, Jr., J. L. Bohn, B. D. Esry, and C. H. Greene, Phys. Rev. Lett. 80, 2097 (1998).

[28] S. J. J. M. F. Kokkelmans and E. G. M. van Kempen, personal communication (unpublished).

[29] J. L. Bohn, personal communication (unpublished).

[30] J. E. Williams, N. Nygaard, and C. W. Clark, condmat/0511011 (2005).

[31] J. F. Bertelsen and K. Mølmer, Phys. Rev. A 73, 013811 (2006).

[32] The atom loss spectrum was recorded for a ${ }^{87} \mathrm{Rb}$ gas with $50 \%$ condensate fraction and two non-condensed gases with temperatures of $130 \mathrm{nK}$ and $190 \mathrm{nK}$. Over this range in temperature the measured resonant frequency shifts by only $5(2) \mathrm{Hz} / \mathrm{nK}$. The small temperature dependence that we observe can be explained by the fact that the molecule creation process preferentially selects atom pairs with low relative momentum and there exists an abundance of pairs available for each ${ }^{85} \mathrm{Rb}$ atom in the gas.

[33] J. P. Burke and J. L. Bohn, Phys. Rev. A 59, 1303 (1999).

[34] D. S. Jin, posters at DAMOP and ICAP in 2006 (unpublished).

[35] C. Ospelkaus et al., cond-mat/0607581 (2006). 


\title{
Observation of Heteronuclear Feshbach Molecules from $\mathbf{a}^{85} \mathrm{Rb}-{ }^{87} \mathrm{Rb}$ gas
}

\author{
S. B. Papp* and C. E. Wieman \\ JILA, National Institute of Standards and Technology and University of Colorado, Boulder, Colorado 80309-0440, USA
}

(Dated: August 27, 2018)

\begin{abstract}
We report on the observation of ultracold heteronuclear Feshbach molecules. Starting with a ${ }^{87} \mathrm{Rb} \mathrm{BEC}$ and a cold atomic gas of ${ }^{85} \mathrm{Rb}$, we utilize previously unobserved interspecies Feshbach resonances to create up to 25,000 molecules. Even though the ${ }^{85} \mathrm{Rb}$ gas is non degenerate, we observe a large molecular conversion efficiency due to the presence of a quantum degenerate ${ }^{87} \mathrm{Rb}$ gas; this represents a key feature of our system. We compare the molecule creation at two different Feshbach resonances with different magnetic-field widths. The two Feshbach resonances are located at $265.44 \pm 0.15 \mathrm{G}$ and $372.4 \pm 1.3 \mathrm{G}$. We also directly measure the small binding energy of the molecules through resonant magnetic-field association.
\end{abstract}

The creation of ultracold molecules from ultracold atoms is currently a topic of great experimental and theoretical interest [? ? ? ? ]. Ultracold heteronuclear molecules in low-lying vibrational states are particularly interesting since they are predicted to exhibit a permanent dipole moment due to the unequal distribution of electrons. Numerous proposals for utilizing polar molecules exist, including quantum computation [? ] and the search for the electron electric dipole moment [? ]. Although no significant permanent dipole moment is expected to exist in a ${ }^{85} \mathrm{Rb}-{ }^{87} \mathrm{Rb}$ molecule, our work demonstrates a first step toward the efficient production of ground-state ultracold heteronuclear molecules.

To date cold heteronuclear molecules in high-lying vibrational levels have been created using photoassociation [? ? ]. These molecules can then be pumped toward low-lying vibrational levels by exciting boundbound molecular transitions [? ] via, for example, stimulated Raman-type transitions [? ] that enhance the probability of populating the lowest vibrational level. The initial photoassociation step used in this process is inefficient, and many final vibrational levels of the molecule are occupied. An alternative to this initial photoassociation step is the direct conversion of two free atoms into a molecule in the highest vibrational levels using a Feshbach resonance [? ? ? ? ? ? ? ? . High conversion efficiency using a Feshbach resonance has been demonstrated in single-species gases via adiabatic magneticfield sweeps across the resonance [? ? ], three-body recombination [? ], resonant magnetic-field association [? ], and non adiabatic magnetic-field sweeps [? ? ]. Feshbach resonances between two different atomic species [? ? ] have previously been reported. Our work builds upon these observations by demonstrating stable heteronuclear Feshbach molecules.

In this Letter we present a systematic study of the creation of heteronuclear molecules from an atomic gas of ${ }^{85} \mathrm{Rb}$ and ${ }^{87} \mathrm{Rb}$. We find that these molecules can be created using the standard techniques already proven with single-species gases. The presence of two species with different quantum degeneracy provides a rich system for testing our understanding of the conversion efficiency from atoms to molecules. Furthermore, the molecule creation process allows us to determine the location and width of Feshbach resonances in the two-species system; this information will be required for future studies of the ${ }^{85} \mathrm{Rb}-{ }^{87} \mathrm{Rb}$ system with a tunable interspecies interaction.

Our apparatus is designed to create a two-species Bose gas through sympathetic cooling of ${ }^{85} \mathrm{Rb}$ with ${ }^{87} \mathrm{Rb}$. The details of our system are similar to those described in Refs. [? ? ] and are only briefly described here. We initially collect approximately $3 \times 10^{9}{ }^{87} \mathrm{Rb}$ atoms and $10^{7}$ ${ }^{85} \mathrm{Rb}$ atoms in a two-species vapor cell magneto-optical trap (MOT). The atoms are loaded into a quadrupole magnetic trap with a $100 \mathrm{G} / \mathrm{cm}$ magnetic-field gradient in the axial direction of the coils and physically transported to another region of the apparatus with lower vacuum pressure [? ? ]. Here the atoms are prepared in the ${ }^{85} \mathrm{Rb}$ $\left|f=2, m_{f}=-2\right\rangle$ state and ${ }^{87} \mathrm{Rb}\left|f=1, m_{f}=-1\right\rangle$ state and loaded into a Ioffe-Pritchard-type magnetic trap. Selective $\mathrm{rf}$ evaporation is used to lower the temperature of the ${ }^{87} \mathrm{Rb}$ gas, while the ${ }^{85} \mathrm{Rb}$ gas is sympathetically cooled through thermal contact with the ${ }^{87} \mathrm{Rb}[?]$.

The mixed-species gas is evaporatively cooled in the magnetic trap to approximately $10 \mu \mathrm{K}$ and then further evaporatively cooled in an optical dipole trap. The optical trap is formed at the focus of a Yb:YAG laser beam with a $1 / e^{2}$ radius of $23.5 \mu \mathrm{m}$. To evaporate in the optical trap the power is lowered from $1.2 \mathrm{~W}$ to $14 \mathrm{~mW}$ in 10 seconds. This trap has the distinct advantage of holding any spin state in the same spatial location while allowing a variable magnetic field to be applied. A Helmholtz pair of coils provides a magnetic field up to $700 \mathrm{G}$; the magnetic field is calibrated using rf-driven Zeeman transitions with a systematic uncertainty of $0.01 \%$. We perform the optical trap evaporation slightly above the ${ }^{85} \mathrm{Rb}$ Feshbach resonance at $155 \mathrm{G}$ where density-dependent ${ }^{85} \mathrm{Rb}-$ ${ }^{85} \mathrm{Rb}$ loss is minimized [? ]. We typically produce a ${ }^{87} \mathrm{Rb}$ BEC with 300,000 atoms and 50\% condensate fraction and a non degenerate $\left(\mathrm{T} / \mathrm{T}_{c}=2.4\right)$ gas of ${ }^{85} \mathrm{Rb}$ with 40,000 atoms. Measurements are performed in an optical trap with a radial trap frequency of $\omega_{r}=2 \pi \times 200$ $\mathrm{Hz}$ and an aspect ratio of approximately 100. 


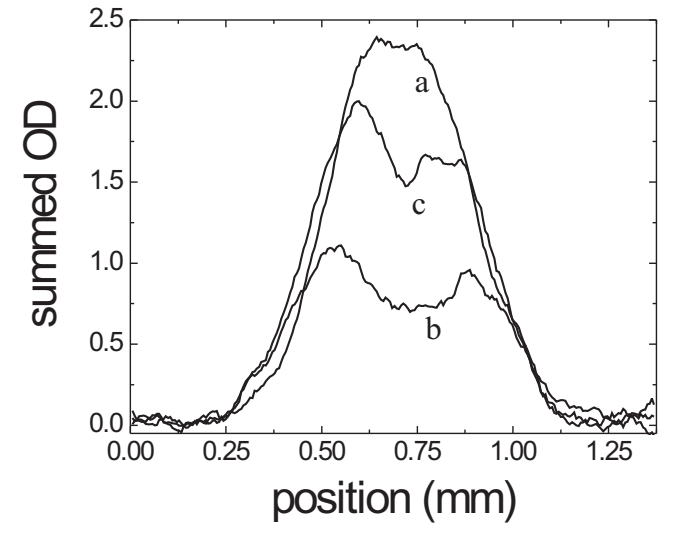

FIG. 1: Absorption-image axial cross sections of the ${ }^{85} \mathrm{Rb}$ gas demonstrating reversible molecule creation. The measured two-dimensional optical density (OD) was summed in the remaining radial direction of the absorption image. (a) Prior to sweeping the magnetic field the atom number is 26,500. (b) After sweeping through the resonance $53 \%$ of the gas is converted to molecules. (c) By reversing the molecule creation process approximately $85 \%$ of the initial atom number is observed to reappear. Note that most of the ${ }^{85} \mathrm{Rb}$ loss occurs in the center of the gas where the ${ }^{87} \mathrm{Rb} \mathrm{BEC}$ density is largest.

We have discovered two heteronuclear $s$-wave Feshbach resonances in the ${ }^{85} \mathrm{Rb}\left|f=2, m_{f}=-2\right\rangle$ state and ${ }^{87} \mathrm{Rb}$ $\left|f=1, m_{f}=-1\right\rangle$ state, one near $265 \mathrm{G}$ and the other near $372 \mathrm{G}$. The binding energy of the bound molecular state increases with magnetic field above the position of each Feshbach resonance. To create heteronuclear molecules we adiabatically $(450 \mu \mathrm{s} / \mathrm{G})$ sweep [? ] the magnetic field from low to high field through a Feshbach resonance. Figure 10 shows an absorption-image cross section of the ${ }^{85} \mathrm{Rb}$ gas following a sweep through the resonance. Prior to imaging the gas is released from the optical trap and expands for $3 \mathrm{~ms}$; at the same time as the optical trap release is initiated, the magnetic field is switched off within $50 \mu \mathrm{s}$. A significant fraction $(\sim 60 \%)$ of the atoms disappear as compared to an absorptionimage cross section without sweeping the magnetic field (Fig. [1). When we reverse the molecule creation process by immediately applying a second field sweep in the opposite direction less than $100 \mu$ s after the first sweep ends, (Fig. 15), a large fraction of the atoms reappear [? ]. The atoms that reappear represent reversible heteronuclear molecule formation. The small fraction of atoms that do not return are lost to ${ }^{85} \mathrm{Rb}-{ }^{87} \mathrm{Rb}$ inelastic collisions that remove atoms from the trap. We verified this by sweeping across the resonance at the same rate in the opposite direction. At a typical ${ }^{87} \mathrm{Rb}$ peak density of $1 \times 10^{14} \mathrm{~cm}^{-3}$ we observe approximately $15 \%{ }^{85} \mathrm{Rb}$ number loss during a sweep. By monitoring the reappearance
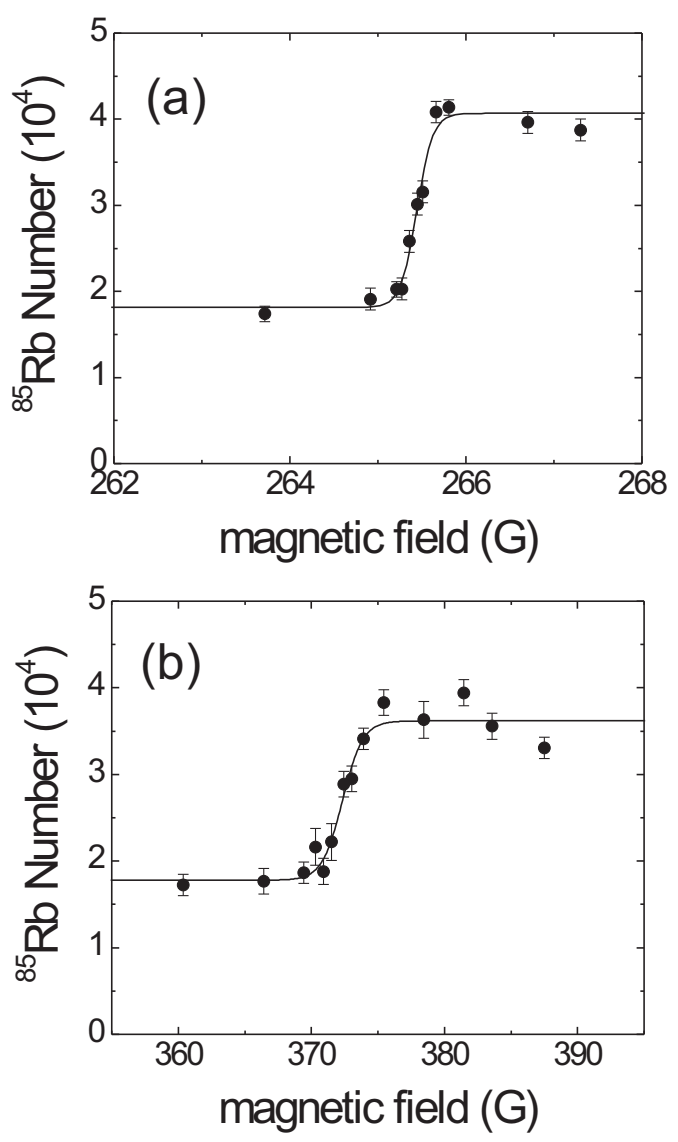

FIG. 2: Atom loss after magnetic-field sweeps through a Feshbach resonance. The number of ${ }^{85} \mathrm{Rb}$ atoms is a function of the final magnetic field during the sweeps near the (a) 265 $\mathrm{G}$ and (b) $372 \mathrm{G}$ Feshbach resonances. Initially there are $2.1-2.3 \times 10^{5}{ }^{87} \mathrm{Rb}$ atoms at $\mathrm{T} / \mathrm{T}_{c}$ between 0.82 and 0.84 . The data are fitted to an error function to extract the center position and width. The resulting positions of the two transitions are $265.44 \pm 0.15 \mathrm{G}$ and $372.4 \pm 1.3 \mathrm{G}$ with the uncertainty given by the fitted RMS width.

of atoms as a function of time, we find that the molecules decay with a lifetime of approximately $1 \mathrm{~ms}$. This decay is due to a combination of inelastic collisions with the ${ }^{87} \mathrm{Rb}$ gas and a one-body spontaneous process [? ? ].

We precisely located the Feshbach resonances with an experimental technique that avoids the need for rapid magnetic-field sweeps. At the lower (higher) field resonance we sweep the magnetic field from 269 G (397 G) downward toward the resonance stopping at various final values. The rate of this magnetic field sweep is fast compared to both the timescale for molecule creation and atom loss due to inelastic collisions. The field is held at the final value for $0.3 \mathrm{~ms}$ and then returns to $269 \mathrm{G}$ (397 
G) at a rate of $450 \mu \mathrm{s} / \mathrm{G}(70 \mu \mathrm{s} / \mathrm{G})$ which is slow compared to the molecule creation rate. The magnetic field remains here for $5 \mathrm{~ms}$ to ensure that any molecules made during the second sweep decay and are lost from the optical trap [? ? ]. We then simultaneously turn off the magnetic field and the optical trap to let the gas expand for $6 \mathrm{~ms}$ and measure the number of ${ }^{85} \mathrm{Rb}$ atoms remaining. In Fig. 2 we show the atom number remaining as a function of the final magnetic field for the two Feshbach resonances. The key to this method is that if the field passes through the Feshbach resonance on the first sweep, then a fraction of the atoms will be converted into molecules by the second sweep. The rapid onset of atom loss due to molecule creation when the magnetic field is swept below $265.44 \mathrm{G}$ (372.4 G) represents crossing the peak of the Feshbach resonance. A significant fraction $(\sim 50 \%)$ of the ${ }^{85} \mathrm{Rb}$ gas is converted to molecules.

The dependence of molecule conversion efficiency on sweep rate was measured in Ref. [? ] and is well characterized by a Landau-Zener model. For our two-species gas with similar number and temperature we observe a factor of $5.3 \pm 1.9$ decrease in the ramp rate required to create molecules at the $372 \mathrm{G}$ Feshbach resonance as compared to the $265 \mathrm{G}$ resonance. This factor is consistent with the ratio of the predicted widths of the two resonances, which is in the range of $6-8[?$ ? ? ]. This directly verifies the predicted inverse relationship between the Feshbach resonance width and the sweep rate required to create molecules.

We have investigated the adiabatic conversion efficiency of atoms to molecules. We begin by sweeping the magnetic field upward through the 265 G Feshbach resonance at a rate that is slow with respect to the molecule conversion rate. It is then held at $269 \mathrm{G}$ for $5 \mathrm{~ms}$ to allow the molecules to decay and be lost from the trap. The number of molecules formed is simply the difference in ${ }^{85} \mathrm{Rb}$ number before and after the sweep with a small correction applied to account for measured inelastic atom loss during the field sweeps. The conversion efficiency to heteronuclear molecules is shown in Fig. 3 as a function of ${ }^{87} \mathrm{Rb} \mathrm{T} / \mathrm{T}_{c}$. We varied $\mathrm{T} / \mathrm{T}_{c}$ by either changing the number of ${ }^{87} \mathrm{Rb}$ atoms or the temperature. The ${ }^{85} \mathrm{Rb}$ gas had a $\mathrm{T} / \mathrm{T}_{c}$ in the range of 2.2 to 4 . We observe up to $60 \%$ conversion of the ${ }^{85} \mathrm{Rb}$ gas into molecules even when the ${ }^{85} \mathrm{Rb}$ gas is far from quantum degeneracy. The largest conversion efficiency is observed when ${ }^{85} \mathrm{Rb}$ is least quantum degenerate, indicating that the conversion efficiency primarily depends on ${ }^{87} \mathrm{Rb} \mathrm{T} / \mathrm{T}_{c}$.

In a single-species gas the molecule conversion efficiency of an adiabatic field sweep was shown to depend only on the degree of quantum degeneracy [? ? ]. A molecule is formed during an adiabatic sweep if two free atoms are sufficiently close in phase space so that their wavefunction can smoothly evolve to a bound molecule as the Feshbach resonance is crossed. This model also explains the behavior we observe here. Each particle of

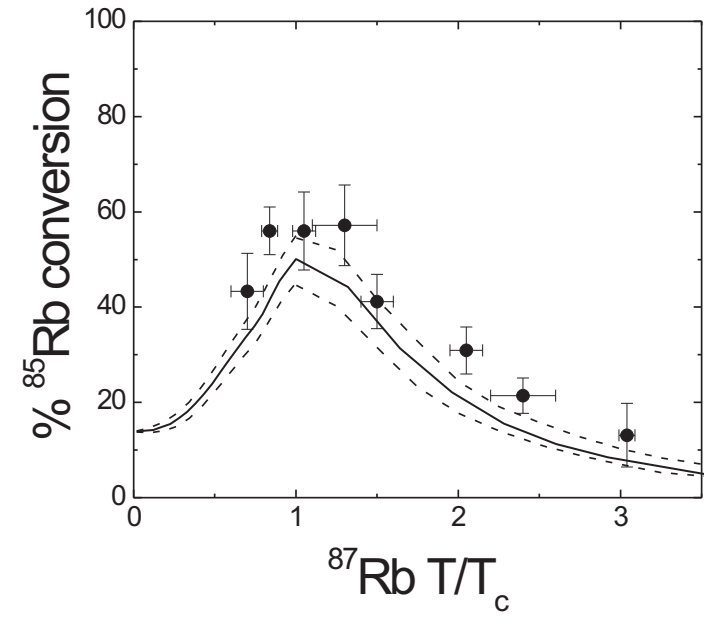

FIG. 3: Heteronuclear molecule conversion efficiency at the $265 \mathrm{G}$ Feshbach resonance as a function of ${ }^{87} \mathrm{Rb} \mathrm{T} / \mathrm{T}_{c}$. At our largest conversion efficiency the ${ }^{85} \mathrm{Rb}$ gas has $\mathrm{T} / \mathrm{T}_{c}=2.6$. The solid line shows a simulation based on our conversion model, and the dashed lines represent the uncertainty. The conversion drops below $\mathrm{T} / \mathrm{T}_{c}=1$ since the $\mathrm{BEC}$ is spatially smaller than the thermal ${ }^{85} \mathrm{Rb}$ gas and the conversion process depends on the proximity of two atoms in phase space.

the less degenerate species is surrounded in phase space by particles of the more degenerate ${ }^{87} \mathrm{Rb}$ gas with which molecules can be formed. We used a Monte-Carlo simulation to model the observed conversion efficiency. Phase space distributions of position and momenta of ${ }^{85} \mathrm{Rb}$ and ${ }^{87} \mathrm{Rb}$ are randomly generated based on the number and temperature of each gas and the trap frequencies. For the ${ }^{87} \mathrm{Rb}$ gas at temperatures above $\mathrm{T}_{c}$ a MaxwellBoltzmann (MB) distribution is used. Below $\mathrm{T}_{c}$ we use a combination of a Thomas-Fermi distribution for the BEC and a Bose-Einstein distribution for the thermal component. A MB distribution is always used for the ${ }^{85} \mathrm{Rb}$ gas since the temperature of the gas does not fall below $\mathrm{T}$ $=2.2 \mathrm{~T}_{c}$. For each ${ }^{85} \mathrm{Rb}$ atom the simulation searches the ${ }^{87} \mathrm{Rb}$ gas to find a pair that is sufficiently close in phase space. After a pairing occurs, the two atoms are removed from the simulation. Two atoms are considered sufficiently close in phase space if the following conditions are met: (1) If a BEC is present, any ${ }^{85} \mathrm{Rb}$ atoms inside the Thomas Fermi radius of the condensate form a molecule, and (2) outside the BEC, an ${ }^{85} \mathrm{Rb}$ atom and a partner ${ }^{87} \mathrm{Rb}$ atom must satisfy the relation found in Ref. [? ]: $\left|\delta r_{\text {rel }} m \delta v_{\text {rel }}\right|<\gamma h$, where $\delta r_{r e l}$ is the separation of the pair, $m$ is the atomic mass, $\delta v_{r e l}$ is the relative velocity, and $\gamma=0.44 \pm 0.03$.

The results of our simulation are shown by the solid line in Fig. 3. For $\mathrm{T} / \mathrm{T}_{c}>1$ each gas has roughly 
the same spatial size in the optical trap, and therefore molecule conversion can occur anywhere in the gas as long as the local phase space criterion is met. At $\mathrm{T} / \mathrm{T}_{c}=1$ and below, a significant fraction of the ${ }^{87} \mathrm{Rb}$ atoms are part of the condensate, which is spatially small compared to the extent of the ${ }^{85} \mathrm{Rb}$ gas. If the ${ }^{85} \mathrm{Rb}$ gas were a BEC, our simulation would predict that the molecule conversion efficiency quickly approaches $100 \%$ as the $\mathrm{T} / \mathrm{T}_{c}$ of each gas drops below one. There is good agreement between the experiment and simulation in both the non degenerate and quantum degenerate regimes, suggesting that the pairing model in Ref. [? ] is also applicable to heteronuclear molecule creation.

We created heteronuclear molecules using a small oscillating magnetic field to do spectroscopy on the Feshbach bound state. The oscillating field causes two atoms to bind together and form a molecule [? ? ]. We first ramp the magnetic field from $269 \mathrm{G}$ to a selected value between 266.2 and $267 \mathrm{G}$ in $0.3 \mathrm{~ms}$. The field modulation is then applied using the Helmholtz coil pair for $20 \mathrm{~ms}$ with a peak-to-peak amplitude between 0.6 and $1.0 \mathrm{G}$ at a frequency up to $40 \mathrm{kHz}$. Next the field is returned to 269 $\mathrm{G}$ and held there for $5 \mathrm{~ms}$, allowing any molecules made during the modulation to decay. Finally we determine the number of atoms remaining as before. As in Ref. [? ], we observe strongly enhanced atom loss at certain frequencies, and the loss depends on the duration and amplitude of the modulation. A typical loss spectrum is shown in the inset of Fig. 4 the frequency at which we observe maximum atom loss gives a measure of the binding energy of the molecules [?].

The resonant frequency of maximum atom loss is shown in Fig. \$ for various magnetic fields near the Feshbach resonance. For each loss curve the amplitude used converted roughly half the ${ }^{85} \mathrm{Rb}$ gas into molecules. The solid line is a fit to the data based on the universal form of the molecular binding energy near an $s$-wave Feshbach resonance [? ]. In the fit the background scattering length is fixed to the value $213 \pm 7 a_{0}$ [? ], and the Feshbach-resonance peak position and width are varied; the best fit finds the peak position and width to be $265.42 \pm 0.08 \mathrm{G}$ and $5.8 \pm 0.4 \mathrm{G}$, respectively. These results are consistent with our previously discussed determination of the Feshbach-resonance peak position and with the predicted width of the Feshbach resonance in Refs. [? ? ? ].

In summary, we have created heteronuclear Feshbach molecules from an ultracold gas of ${ }^{85} \mathrm{Rb}$ and ${ }^{87} \mathrm{Rb}$. We demonstrated that molecules can be produced with two methods, magnetic-field sweeps and resonant-field modulation. The conversion efficiency of ${ }^{85} \mathrm{Rb}$ into molecules can reach $60 \%$ even when that gas is not quantum degenerate. The heteronuclear molecules described here are ultracold and are stable for at least $1 \mathrm{~ms}$. These conditions may provide a first step toward the efficient production of ground-state heteronuclear molecules.

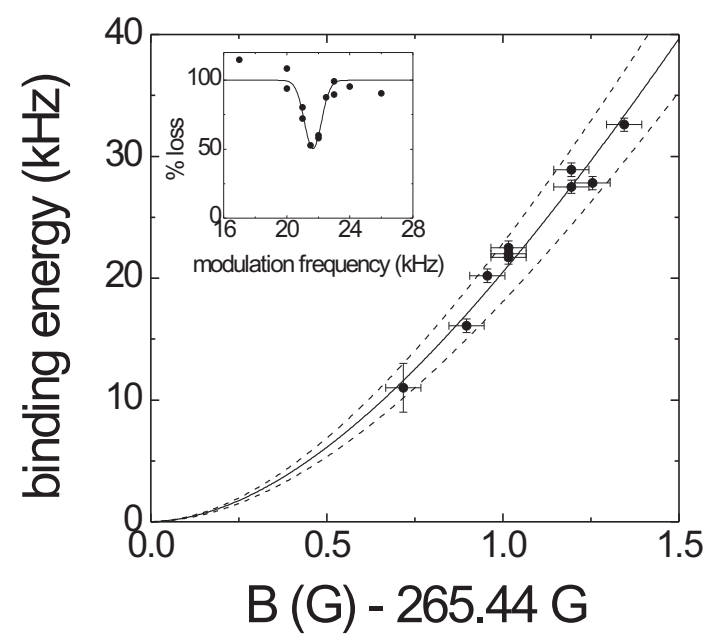

FIG. 4: Resonant frequency of atom loss as a function of magnetic field. The solid line is a fit to the data based on the universal binding energy of $s$-wave Feshbach molecules and the dashed lines represent the uncertainty in the Feshbach resonance width. (Inset) An atom-loss spectrum at $266.5 \mathrm{G}$ as a function of the modulation frequency. The modulation converts roughly $50 \%$ of the gas to molecules. The loss is centered at $21.7 \mathrm{kHz}$ with a width of $0.6 \pm 0.2 \mathrm{kHz}$. The solid line is a gaussian fit to the data. We report the uncertainty in the binding energy as the width of the loss spectrum because we lack a detailed understanding of the lineshape.

Recently evidence of heteronuclear molecule creation has been reported using ${ }^{40} \mathrm{~K}$ and ${ }^{87} \mathrm{Rb}$ in Refs. [? ? ].

We gratefully acknowledge useful discussions with Debbie Jin, John Bohn, Servaas Kokkelmans, and Cindy Regal. We thank Josh Zirbel and Juan Pino for experimental assistance. S. B. P acknowledges support from an NSF Graduate Fellowship. This work has been supported by NSF and ONR.

* Email: papp@jilau1.colorado.edu 